\title{
Nanomedicine: Development and Challenges in Cancer Treatment
}

\author{
Jaidip Jagtap ${ }^{1}$, Amit Joshi ${ }^{1}$, Nrusingh C. Biswal ${ }^{2 *}$ \\ ${ }^{1}$ Department of Biomedical Engineering, Medical College of Wisconsin, Milwaukee, WI 53226, USA \\ ${ }^{2}$ Department of Radiation Oncology, University of Maryland School of Medicine, Baltimore, MD 21201, USA
}

*Corresponding author: Nrusingh C Biswal, Department of Radiation Oncology, University of Maryland School of Medicine, Baltimore, MD 21201, USA.

To Cite This Article: Nrusingh C. Biswal, Nanomedicine: Development and Challenges in Cancer Treatment. Am J Biomed Sci \& Res. 2019 - 6(3). AJBSR.MS.ID.001036. DOI: 10.34297/AJBSR.2019.06.001036.

Received: 眥 October 01, 2019; Published: 眥 November 25, 2019

\begin{abstract}
A brief review of the literatures on development and challenges associated with success of nanomedicine for cancer therapy is presented. Nanomaterial shape, size and characteristics plays an important role in an effective treatment. The chemotoxicity, non-targeted and non-uniform heating in tumors were the major drawbacks in nanomedicine assisted hyperthermia, however recent developments in nanomaterials possessing multifunctional characteristics enabled better diagnosis and helped in the treatment of cancer.
\end{abstract}

Keywords: Nanoparticles; Gold nanoparticles; Liposomes; Carbon nanoparticles; Fluorescence imaging; Quantum dots

\section{Introduction}

Radiation therapy has been successfully applied in clinics for cancer therapy; however, its success rate is limited to $\sim 50 \%$ due to non-responsive cancer cells for therapy alone and it's very expensive. Chemotherapy uses chemicals to kill or stop the growth of cancer cells however it also damages the fast-growing healthy cells. Nanoparticle has shown great potential along with radiation therapy or chemotherapy for improved treatment response. The major setback for nanoparticle assisted hyperthermia for cancer therapy was non-specific targeted, uncontrolled drug release, nonuniform heating of tumor result in relapsing the tumor post therapy, biocompatibility and nanomaterial toxicity concerns. However, the continuous development for smart nanoparticles being in progress for possessing increased drug loading capacity, externally triggering for localized delivery, multimodal imaging contrast agent enabled tumor diagnosis and post therapy monitoring the effect. Liposomes, iron-oxide, gold are widely used nanoparticles for various applications in nanomedicine and further quantum dots, micelles, dendrimers and meso-porous silica nanoparticles have been receiving attention due to the context of their structures, biocompatibility, and functionality as carrier and deliver at site. Apart from inorganic material, FDA approved organic dye (Indocyanine green) has also been utilized for real time imaging to discriminate genetically modified tumor microenvironment [1], exploring in near infrared (NIR-II) imaging window for depth imaging [2] and as a therapeutic agent [3,4] but it doesn't help in conjugating antibody on it or targeting tumor.

Conventional limitations on liposomes use had been revolutionized by PEGylation helped in longer circulation times in the blood; targeting cancer cell achieved by conjugating with monoclonal antibodies, proteins, glycoproteins, peptides, antibody fragments, vitamins and carbohydrates [5-8]; controlled drug release by external stimuli of ultrasound [9], and microwaves field [10]; functionalized radiolabeled liposomes [11-13] serving both imaging as well as therapeutics [11]. Meso-porus silica nanoparticles can be tuned to size $5-50 \mathrm{~nm}$ diameter for utilizing drug loading capacity, drug delivery and further as a dual imaging contrast agent in fluorescence and magnetic resonance imaging [14]. Polymer coated super paramagnetic iron oxide nanoparticles (SPIONs) can be controlled by an external magnetic field for accumulation at region of interest, deliver drugs, magnetic resonance imaging 
and magnetic hyperthermia therapy for tumor [15-17]. Carbon nanotubes can be used in form of single wall or multi wall carbon nanotubes which strongly absorbs NIR wavelengths and are also a strong candidate for photo thermal ablation. Functionalized carbon nanotubes demonstrated potential as carrier for siRNA, DNA [18]; crosses the blood-brain barrier [19,20]; and can be used as a therapeutic agent in photothermal ablation for cancer treatment [21]. Hangi dai and his group had demonstrated single walled carbon nanotube for effectively imaging the brain vasculature which could help in accessing damaged site [22]. Quantum dots (QDs) are semiconducting nanomaterial demonstrated for use as fluorescence imaging, adaptability for conjugating antibody and biocompatible for in vivo imaging [23]. QDs provide fluorescence imaging in $700-1300 \mathrm{~nm}$ wavelength range allowing $\sim 0.5-1 \mathrm{~cm}$ depth tumor imaging and can also be conjugated for targeting human prostate cancer in mice cancer [24]. Qiangbin Wang group had conjugated polyethylene glycol-coated silver sulfide quantum dots (PEG-Ag2S QDs) with anti-cancer drug doxorubicin and demonstrate significant tumor inhibition with high resolution fluorescence imaging. Further, such studies can be tested for various cancer types.

Photothermal therapy has major drawback of depth penetration and it can be used at surface level skin tumor therapy. Gold nanoparticles mainly used photothermal ablation since they have high absorption at $808 \mathrm{~nm}$ wavelength can efficiently generate heat to increase temperature to kill cancer cells [25]. Gold nanoparticle can be used for drug delivery with external (x-ray, Ultrasound, laser) or internal ( $\mathrm{pH}$, redox reaction) triggering $[26,27]$. To overcome the depth penetration disadvantage recently our group has shown delivering therapeutic nanoparticles at site with catheter and ablating the site with controlled laser carried through fiber via the same catheter for liver cancer therapy at 3-4cm depth in 300-400 g rat [28]. Further the study has shown that the nanoparticle size and shape also play an important role in nanoparticle circulation through blood which impact on successful therapy [29]. Thus, nanoparticle has made progress in increasing long circulation time, adaptability to graft antibody for cancer therapy, increased loading capacity and controlled drug release. There is still a long way to go for improving better control on tumor boundary separation to avoid collateral damage to nearby healthy cells, testing nanoparticles for various cancer types and dose calibration which could help in improving success of nanomedicine for cancer therapy.

\section{References}

1. J Jagtap, G Sharma, A K Parchur, V Gogineni, C Bergom, et al. (2018) Methods for detecting host genetic modifiers of tumor vascular function using dynamic near-infrared fluorescence imaging. Biomed Opt Express $9(2):$ 543-556.

2. J A Carr, D Franke, J R Caram, C F Perkinson, M Saif, et al. (2018) Shortwave infrared fluorescence imaging with the clinically approved near-infrared dye indocyanine green. Proc Natl Acad Sci USA 115(17): 4465-4470.
3. K Hirohashi, T Anayama, H Wada, T Nakajima, T Kato, et al. (2015) Photothermal ablation of human lung cancer by low-power nearinfrared laser and topical injection of indocyanine green. J Bronchology Interv Pulmonol 22(2): 99-106.

4. C Shirata, J Kaneko, Y Inagaki, T Kokudo, M Sato, et al. (2017) Nearinfrared photothermal/photodynamic therapy with indocyanine green induces apoptosis of hepatocellular carcinoma cells through oxidative stress. Sci Rep 7: 13958.

5. G T Noble, J F Stefanick, J D Ashley, T Kiziltepe, B Bilgicer (2014) Ligandtargeted liposome design: challenges and fundamental considerations. Trends Biotechnol 32(1): 32-45.

6. R R Sawant, V P Torchilin (2012) Challenges in development of targeted liposomal therapeutics. AAPS J 14(2): 303-315.

7. E Ruoslahti (2012) Peptides as targeting elements and tissue penetration devices for nanoparticles. Adv Mater 24(28): 3747-3756.

8. P Sapra, T M Allen (2003) Ligand-targeted liposomal anticancer drugs. Prog Lipid Res 42(5): 439-462.

9. S L Huang, R C MacDonald (2004) Acoustically active liposomes for drug encapsulation and ultrasound-triggered release. Biochim Biophys Acta 1665(1-2): 134-141.

10. Y Jin, X Liang, Y An, Z Dai (2016) Microwave-Triggered Smart Drug Release from Liposomes Co-encapsulating Doxorubicin and Salt for Local Combined Hyperthermia and Chemotherapy of Cancer. Bioconjug Chem 27(12): 2931-2942.

11. A L Petersen, AE Hansen, A Gabizon, T L Andresen (2012) Liposome imaging agents in personalized medicine. Adv Drug Deliv Rev 64(13): 1417-1435.

12. I Ogihara Umeda, T Sasaki, S Kojima, H Nishigori (1996) Optimal Radiolabeled Liposomes for Tumor Jmaging J Nucl Med 37: 326-332.

13. S Li, B Goins, L Zhang, A Bao (2012) Novel multifunctional theranostic liposome drug delivery system: construction, characterization, and multimodality MR, near-infrared fluorescent, and nuclear imaging. Bioconjug Chem 23(16): 1322-1332.

14. S Hossen, M K Hossain, M K Basher, M N H Mia, M T Rahman, et al. (2019) Smart nanocarrier-based drug delivery systems for cancer therapy and toxicity studies: A review. J Adv Res 15: 1-18.

15. M Mahmoudi, S Sant, B Wang, S Laurent, T Sen (2011) Superparamagnetic iron oxide nanoparticles (SPIONs): development, surface modification and applications in chemotherapy. Adv Drug Deliv Rev 63(1-2): 24-46.

16.S Laurent, A A Saei, S Behzadi, A Panahifar, M Mahmoudi (2014) Superparamagnetic iron oxide nanoparticles for delivery of therapeutic agents: opportunities and challenges. Expert Opin Drug Deliv 11(9): $1449-1470$

17. P B Santhosh, NP Ulrih (2013) Multifunctional superparamagnetic iron oxide nanoparticles: promising tools in cancer theranostics. Cancer Lett 336(1): 8-17.

18. KH Son, J H Hong, JW Lee (2016) Carbon nanotubes as cancer therapeutic carriers and mediators. Int J Nanomedicine 11: 5163-5185.

19. H Kafa, J T Wang, N Rubio, K Venner, G Anderson, et al. (2015) The interaction of carbon nanotubes with an in vitro blood-brain barrier model and mouse brain in vivo. Biomaterials 53: 437-452.

20.J T Wang, K T Al Jamal (2015) Functionalized carbon nanotubes: revolution in brain delivery. Nanomedicine (Lond) 10(17): 2639-2642.

21. S Y Madani, N Naderi, O Dissanayake, A Tan, A M Seifalian (2011) A new era of cancer treatment: carbon nanotubes as drug delivery tools. Int J Nanomedicine 6: 2963-2979.

22. G Hong, S Diao, J Chang, A L Antaris, C Chen, (2014) Through-skull fluorescence imaging of the brain in a new near-infrared window. Nat Photonics 8(9): 723-730. 
23. L Qi, X Gao (2008) Emerging application of quantum dots for drug delivery and therapy. Expert Opin Drug Deliv 5(3): 263-267.

24. M S Muthu, S A Kulkarni, A Raju, SS Feng (2012) Theranostic liposomes of TPGS coating for targeted co-delivery of docetaxel and quantum dots. Biomaterials 33(12): 3494-3501.

25. I H El Sayed, X Huang, M A El (2005) Sayed Surface plasmon resonance scattering and absorption of anti-EGFR antibody conjugated gold nanoparticles in cancer diagnostics: applications in oral cancer. Nano Lett 5(5): 829-834.

26. C P Yao, L W Zhang, J Wang, Y L He, J Xin, et al. (2016) Recent advances in bismuth-containing photocatalysts with heterojunctions. J Nanomater Pp. 780-791.
27. L Tian, L Lu, Y Qiao, S Ravi, F Salatan, et al. (2016) Stimuli-Responsive Gold Nanoparticles for Cancer Diagnosis and Therapy. J Funct Biomater 7(3): pii. E19.

28. A K Parchur, G Sharma, J M Jagtap, V R Gogineni, P S LaViolette, et al. (2018) Vascular Interventional Radiology-Guided Photothermal Therapy of Colorectal Cancer Liver Metastasis with Theranostic Gold Nanorods. Acs Nano 12(7): 6597-6611.

29. C Ayala Orozco, C Urban, M W Knight, A S Urban, O Neumann, et al (2014) Au Nanomatryoshkas as Efficient Near-Infrared Photothermal Transducers for Cancer Treatment: Benchmarking against Nanoshells. Acs Nano 8(6): 6372-6381. 